\title{
Pros and Cons of Robotic Revisional Bariatric Surgery
}

\author{
Jan Henrik Beckmann ${ }^{a}$ Anne-Sophie Mehdorn ${ }^{a} \quad$ Jan-Niclas Kersebaum ${ }^{a}$ \\ Witigo von Schönfels ${ }^{a}$ Terbish Taivankhuu ${ }^{a}$ Matthias Laudes ${ }^{b}$ \\ Jan-Hendrik Egberts ${ }^{a}$ Thomas Becker ${ }^{a}$ \\ a Department of General, Visceral-, Thoracic-, Transplantation-, and Pediatric Surgery, Kurt-Semm Center for \\ Laparoscopic and Robotic Assisted Surgery, University Hospital Schleswig-Holstein, Campus Kiel, Kiel, Germany; \\ bl. Department of Medicine, University Hospital Schleswig-Holstein, Campus Kiel, Kiel, Germany
}

\section{Keywords}

Obesity · Revisional bariatric surgery · Robotic surgery ·

Roux-en-Y gastric bypass - Laparoscopic surgery ·

Complication

\begin{abstract}
Introduction: Revisional procedures in bariatric surgery are regarded as technically more demanding and riskier than primary interventions. While the use of the surgical robot has not yet proven to be advantageous in primary bariatric interventions, the question remains whether its use is justified for more complex revisional procedures. Objective: To show that revisional bariatric surgery can be performed safely using the da Vinci ${ }^{\circledR}$ Xi surgical system. Methods: We performed a retrospective analysis of prospectively recorded data for revisional bariatric procedures between January 2016 and November 2019. Results: Of 78 revision operations, four (5.1\%) were performed by open surgery, 30 (38.5\%) by laparoscopic surgery, and 44 (56.4\%) by robotic surgery. A comparative analysis of robotic $(n=41)$ versus laparoscopic $(n=$ 18) revisional Roux-en-Y gastric bypasses ( $R$ RYGB) revealed significant differences favoring the robotic approach for operative time (130.7 vs. $167.6 \mathrm{~min}$ ), C-reactive protein values at days 1 (27.9 vs. $49.1 \mathrm{mg} / \mathrm{L}$ ) and 2 (48.2 vs. $83.6 \mathrm{mg} / \mathrm{L}$ ) after surgery, and length of stay (4.9 vs. 6.2 days). Lower complica-
\end{abstract}

karger@karger.com www.karger.com/vis

Karger ${ }^{\prime \prime}=$
C) 2020 S. The Author(s) Published by S. Karger AG, Basel

Karger
Open access

This article is licensed under the Creative Commons AttributionNonCommercial-NoDerivatives 4.0 International License (CC BYNC-ND) (http://www.karger.com/Services/OpenAccessLicense). Usage and distribution for commercial purposes as well as any distribution of modified material requires written permission. tion rates (Clavien-Dindo II-V) were found after rRRYGB (7.3 vs. $22.2 \%$, not significant). Conclusions: Revisional bariatric surgery using a robotic system is safe. The operative time performing $r R R Y G B$ is significantly shorter than $\operatorname{RLYGB}$ in our experience. Otherwise, results were largely comparable. Due to different indications, different index operations and a wide range of revisional procedures, further studies are necessary to confirm these results. @2020 S. The Author(s)

Published by S. Karger AG, Basel

\section{Introduction}

Bariatric surgery is well established in the treatment of morbid obesity. The therapy is highly effective and improves life expectancy and quality of life [1]. However, in addition to the risk of recurrent obesity, there are operative risks and side effects. Due to insufficient weight loss, weight regain, reflux problems, and dysphagia, revisions or conversions are often necessary [2]. Revisional procedures are regarded as technically more demanding and riskier than primary interventions [3]. The indication for revision should be based on a multidisciplinary evaluation and individual risk-benefit assessment, according to current guidelines [4]. Recommended revisional proce- 
dures after adjustable gastric banding (AGB) and sleeve gastrectomy ( $\mathrm{SG}$ ) are primarily Roux-en-Y gastric bypass (RYGB), SG and one anastomosis gastric bypass (OAGB) $[2,5]$.

Robotic systems are increasingly being used in visceral surgery. In addition to three-dimensional, high-definition visualization, intuitive handling with tremor compensation and downscaling, robotic bariatric surgery balances the sometimes massive torque of the abdominal wall. The system seems to be safe in SG [6], but clear advantages are not evident considering higher costs and longer operative times $[7,8]$. With regard to primary RYGB, most studies show that robotic procedures are safe and efficient [9-16]. There are slight advantages in terms of complications, burdened by a longer operative time and higher costs. Differing results from meta-analyses and register studies could result from publication bias and negative effects of the learning curve [17-19].

While primary bariatric interventions generally have a very low complication rate [20], advantages of the robotic system in revision interventions with higher complication rates may be more relevant. So far, there are several reports and case series with good results [21-25] but only a few comparative cohort studies [26, 27]. A low complication rate has been reported, albeit with a low $(n=11)$ number of robotic interventions [26]. Another group found a comparable safety profile of robotic interventions, while robotic revisional surgeries after stapled procedures were performed more quickly than laparoscopic ones [27]. Evaluation of registry databases showed no advantages when using the robot [28]. In particular revisional robotic SG ( $r R S G$ ) showed worse results than $\mathrm{rLSG}$ [29]. A clear benefit is not yet apparent, and it remains unclear whether the use of the surgical robot in revision operations is justified.

We present the largest German series of da $\operatorname{Vinci}^{\circledR} \mathrm{Xi}$ (Intuitive Surgical, Sunnyvale, CA, USA) revisional bariatric procedures, with a comparative analysis of revisional RYGB operations in terms of safety and efficacy.

\section{Materials and Methods}

All bariatric surgeries of the certified obesity center (Referenz zentrum, German Society of General and Visceral Surgery, DGAV) have been prospectively recorded in a database since January 2016. Participating surgeons were accredited as bariatric surgeons by the DGAV. At the time of initiating the evaluation, the department had a cumulative experience of more than 600 bariatric operations (57\% SG, $36 \%$ RYGB, $5 \%$ revisional procedures, and $2 \%$ other). On average, 120 procedures were performed annually between 2013 and 2015. Our department has been using the da Vinci robotic system since 2013. By the end of 2015, 230 robotic procedures were performed within the department, none by the participating surgeons. The first RSG was in 2016, while RYGB procedures were performed robotically from January 2017 [9], using the Xi System from August 2017.

All indications for revisional procedures were made on an interdisciplinary basis. In 2016, only laparoscopic surgery was performed. From mid-2017, the surgical procedure was selected according to availability. There was no specific selection for the use of the robot.

Due to the wide range of revisional procedures, a comparative statistical analysis of all laparoscopic and da Vinci Xi revisional RYGB (rLRYGB and rRRYGB, respectively) procedures was performed. Preoperative parameters included: gender, height, weight, BMI, age, Edmonton Obesity Staging System (EOSS) [30], hemoglobin, white blood cells (WBC), and C-reactive protein (CRP). Operative time, docking time, and intraoperative complications were recorded, as were laboratory values on postoperative days 1 and 2, and length of stay (LOS). In addition to the inpatient course, standardized follow-up data were collected after 1 month and 1 year. The primary endpoint was morbidity after 30 days, according to Clavien-Dindo [31]

Besides a case report of a complex open revision operation [32], a subgroup of 13 rRRYGB operations was published as case series in 2019 [33].

\section{Surgical Techniques}

The different index operations and indications required various surgical procedures. Standard LRYGB and RRYGB have already been described $[9,33]$. The da Vinci Xi robotic platform (Intuitive Surgical, Sunnyvale, CA, USA) was used for all robotic revisional procedures. When performing $\mathrm{RRYGB}$, the pouch was formed using 45-mm linear stapler (Echelon Flex ${ }^{\mathrm{TM}}$ Endopath; Ethicon, Johnson \& Johnson, USA), operated by the assistant surgeon in robotic procedures. The gastrojejunostomy in rRYGB and rOAGB was performed side-to-side using a linear stapler. The enterotomy was closed by a running suture with resorbable material (e.g., Vicryl, Stratafix; Ethicon, Johnson \& Johnson, USA). The jejunojejunostomy in rRYGB was performed similarly. The limb lengths in $\mathrm{rRYGB}$ and $\mathrm{rOAGB}$ varied, depending on the indication for revision. After AGB, the band was usually explanted 6 weeks before final conversion in a two-step procedure. For rSG, a 14-mm bougie was inserted before resection. The proximal staple line was inverted by a continuous suture with Stratafix. For single pouch revision, the pouch was reduced using a linear stapler and the gastrojejunostomy narrowed by a running suture.

\section{Statistical Analysis}

The statistical analyses were performed using SPSS 24 (SPSS Inc., Chicago, IL, USA). Continuous parameters were compared by a two-sided t test, categorical parameters by a $\chi^{2}$ test. A $p$ value $<0.05$ was regarded as statistically significant. Values are presented as mean \pm standard deviation (SD) or $n(\%)$, as appropriate.

\section{Results}

\section{Index Procedure and Indication}

A total of 78 bariatric revisional procedures were performed. The type of revision surgery was dependent on the index operation and revision indication (online suppl. Table 1; for all online suppl. material, see www. karger.com/doi/10.1159/000507742). The most frequent index operation was $S G(n=53,67.9 \%)$, followed by AGB $(n=11,14.1 \%)$, and RYGB $(n=8,10.3 \%)$. Of all SG, one- 
Table 1. Patient characteristics in the revisional (r) laparoscopic (L) and robotic (R) RYGB groups

\begin{tabular}{|c|c|c|c|}
\hline & $\operatorname{rLRYGB}(n=18)$ & rRRYGB $(n=41)$ & $p$ value \\
\hline Mean age $\pm S D$, years & $48.8 \pm 9.2$ & $46.5 \pm 10.3$ & 0.413 \\
\hline $\operatorname{Sex}(\mathrm{f} / \mathrm{m}), n(\%)$ & $11 / 7(61.1 / 38.9)$ & $30 / 11(73.2 / 26.8)$ & 0.354 \\
\hline Mean weight $\pm S D, k g$ & $146.0 \pm 29.7$ & $126.3 \pm 27.5$ & 0.016 \\
\hline Mean height $\pm \mathrm{SD}, \mathrm{cm}$ & $175.6 \pm 13.5$ & $171.3 \pm 9.2$ & 0.169 \\
\hline Mean $\mathrm{BMI} \pm \mathrm{SD}, \mathrm{kg} / \mathrm{m}^{2}$ & $47.1 \pm 6.2$ & $42.9 \pm 8.2$ & 0.061 \\
\hline \multicolumn{4}{|l|}{ EOSS, $n(\%)$} \\
\hline I & $0(0.0)$ & $4(9.8)$ & \\
\hline II & $6(33.3)$ & $18(43.9)$ & \\
\hline III & $10(55.6)$ & $17(41.5)$ & \\
\hline IV & $2(11.1)$ & $2(4.9)$ & 0.340 \\
\hline Mean hemoglobin $\pm \mathrm{SD}, \mathrm{g} / \mathrm{dL}$ & $13.7 \pm 1.9$ & $13.3 \pm 1.5$ & 0.407 \\
\hline Mean $\mathrm{WBC} \pm \mathrm{SD}, 10^{9} / \mathrm{L}$ & $7.4 \pm 2.5$ & $7.3 \pm 2.1$ & 0.894 \\
\hline Mean CRP $\pm S D, m g / L$ & $5.2 \pm 4.9$ & $5.0 \pm 6.9$ & 0.884 \\
\hline \multicolumn{4}{|l|}{ Index operation, $n(\%)$} \\
\hline $\mathrm{AGB}$ & $3(16.7)$ & $2(4.9)$ & \\
\hline VBG & - & $1(2.4)$ & \\
\hline SG & $13(72.2)$ & $36(87.8)$ & \\
\hline OAGB & - & $2(4.9)$ & \\
\hline RYGB & $2(11.1)$ & - & 0.200 \\
\hline \multicolumn{4}{|l|}{ Indication, $n(\%)$} \\
\hline Weight related & $15(83.3)$ & $24(58.5)$ & \\
\hline Other & $3(16.7)$ & $17(41.5)$ & 0.064 \\
\hline
\end{tabular}

Continuous parameters were compared using the two-sided $t$ test and categorical parameters using the $\chi^{2}$ test. A $p$ value $<0.05$ was considered statistically significant (in bold). AGB, adjustable gastric banding; CRP, C-reactive protein; EOSS, Edmonton Obesity Staging System; OAGB, one anastomosis gastric bypass; SD, standard deviation; SG, sleeve gastrectomy; VBG, vertical banded gastroplasty; WBC, white blood cells.

third was converted as a planned second step, a third due to insufficient weight loss or weight gain, and a third due to reflux symptoms. In one case, a chronic and refractory fistula required robotic conversion to RYGB. After AGB, all revision surgeries were performed due to insufficient weight loss or weight regain. After RYGB, revisions were required because of weight regain $(n=2)$, reflux problems $(n=1)$, pouch dilatation $(n=3)$, refractory hypoglycemia $(n=1)$, and gastrogastric fistula $(n=1)$. Six further revisions were performed following vertical banded gastroplasty, OAGB, and biliopancreatic diversion.

\section{Revisional Procedures}

Of 78 revision operations, four (5.1\%) were performed by open surgery, 30 (38.5\%) by laparoscopic surgery, and $44(56.4 \%)$ by robotic surgery. rRYGB was performed most frequently $(n=62,79.5 \%)$, followed by $\mathrm{rSG}$ and $\mathrm{rO}$ AGB ( $n=4$ each, 5.1\%). In individual cases, a hiatal hernia repair and/or a cholecystectomy were performed in parallel. Baseline characteristics are listed in online supplementary Table 2.

Results are shown in online supplementary Table 3. There were no intraoperative complications and no conversions resulting from intraoperative difficulties. Two robotically planned rRYGB procedures were performed openly after initial laparoscopy and evidence of severe adhesions (classified as primarily open). There was no mortality in the postoperative course. One leak of the gastrojejunostomy occurred after open revision of a complex gastrogastric fistula after external RYGB. Six patients underwent postoperative reoperation due to insufficiency $(n=1)$ or hemorrhage $(n=4)$; one re-laparoscopy due to elevated CRP levels after revisional LSG remained without evidence of any cause. We also found one hematoma of the abdominal wall requiring transfusion. In another case, gastrointestinal bleeding occurred but stopped spontaneously, and did not require endoscopy or transfusion. Lower complication rates and shorter surgery times were found after robotic revision surgery (online suppl. Table 3). For details on all cases, see online supplementary Table 4 (raw data).

\section{Laparoscopic versus Robotic rRYGB}

Due to the very different procedures and indications, a comparative analysis of all minimally invasive RYGB revision operations was performed. Table 1 shows the characteristics of 18 rLRYGB versus 41 rRRYGB patients.

While the two groups were largely comparable regarding age, sex, height, comorbidities, and preopera- 
Table 2. Operative parameters and 30-day complication rates between revisional (r) laparoscopic (L) and robotic (R) RYGB

\begin{tabular}{|c|c|c|c|c|}
\hline & $n^{\mathrm{a}}$ & rLRYGB & rRRYGB & $p$ value \\
\hline Mean operative time $\pm \mathrm{SD}$, min & $18 / 41$ & $167.6 \pm 33.8$ & $130.7 \pm 40.4$ & 0.001 \\
\hline Mean docking time $\pm \mathrm{SD}$, min & $-/ 41$ & - & $5.1 \pm 2.9$ & - \\
\hline \multicolumn{5}{|l|}{ Mean hemoglobin \pm SD, g/dL } \\
\hline Day 1 & $18 / 41$ & $11.5 \pm 2.1$ & $11.7 \pm 1.5$ & 0.614 \\
\hline Day 2 & $17 / 41$ & $11.0 \pm 2.5$ & $11.6 \pm 1.5$ & 0.310 \\
\hline \multicolumn{5}{|l|}{ Mean white blood cells $\pm \mathrm{SD}, 10^{9} / \mathrm{L}$} \\
\hline Day 1 & $18 / 40$ & $10.3 \pm 3.6$ & $9.7 \pm 2.5$ & 0.458 \\
\hline Day 2 & $17 / 41$ & $8.0 \pm 2.0$ & $8.1 \pm 2.3$ & 0.867 \\
\hline \multicolumn{5}{|l|}{ Mean CRP $\pm S D, m g / L$} \\
\hline Day 1 & $18 / 39$ & $49.1 \pm 28.7$ & $27.9 \pm 17.6$ & 0.001 \\
\hline Day 2 & $17 / 41$ & $83.6 \pm 49.9$ & $48.2 \pm 36.4$ & 0.004 \\
\hline Mean length of stay $\pm S D$, days & $18 / 41$ & $6.2 \pm 1.6$ & $4.9 \pm 1.0$ & 0.001 \\
\hline Clavien-Dindo classification, $n(\%)$ & $18 / 41$ & & & \\
\hline 0 & & $13(72.2)$ & $37(90.2)$ & \\
\hline I & & $1(5.6)$ & $1(2.4)$ & \\
\hline II & & $2(11.1)$ & $2(4.9)$ & \\
\hline IIIa & & - & - & \\
\hline IIIb & & $2(11.1)$ & $1(2.4)$ & \\
\hline IVa & & - & - & \\
\hline $\mathrm{IVb}$ & & - & - & \\
\hline $\mathrm{V}$ & & - & - & 0.333 \\
\hline Clavien-Dindo II-V, $n(\%)$ & $18 / 41$ & $4(22.2)$ & $3(7.3)$ & 0.103 \\
\hline Reoperations, $n(\%)$ & $18 / 41$ & $2^{b}(11.1)$ & $1^{\mathrm{b}}(2.4)$ & 0.163 \\
\hline Leakage, $n(\%)$ & $18 / 41$ & $0(0.0)$ & $0(0.0)$ & \\
\hline Stenosis, $n(\%)$ & $18 / 41$ & $0(0.0)$ & $0(0.0)$ & \\
\hline Hemorrhage, $n(\%)$ & $18 / 41$ & $3^{c}(16.7)$ & $2^{\mathrm{d}}(4.9)$ & 0.134 \\
\hline Wound infection, $n(\%)$ & $18 / 41$ & $0(0.0)$ & $0(0.0)$ & \\
\hline Pneumonia, $n(\%)$ & $18 / 41$ & $0(0.0)$ & $1(2.4)$ & 0.504 \\
\hline Thrombosis, $n(\%)$ & $18 / 41$ & $1(5.6)$ & $0(0.0)$ & 0.128 \\
\hline Mean excess weight loss $\pm \mathrm{SD}, \mathrm{kg}$ & $18 / 41$ & & & \\
\hline 30 days & $16 / 37$ & $19.2 \pm 10.0$ & $14.7 \pm 7.4$ & 0.070 \\
\hline & $10 / 17$ & $54.4 \pm 29.2$ & $38.0 \pm 16.7$ & 0.073 \\
\hline \multicolumn{5}{|l|}{ Mean BMI change $\pm \mathrm{SD}, \mathrm{kg} / \mathrm{m}^{2}$} \\
\hline 30 days & $16 / 37$ & $-3.9 \pm 1.5$ & $-2.5 \pm 1.5$ & 0.003 \\
\hline 1 year & $10 / 17$ & $-10.9 \pm 4.3$ & $-7.5 \pm 4.6$ & 0.074 \\
\hline
\end{tabular}

Continuous parameters were compared using the two-sided $t$ test and categorical parameters using the $\chi^{2}$ test. A p value $<0.05$ was considered statistically significant (in bold). ${ }^{a}$ Laparoscopic/robotic RYGB groups. ${ }^{b}$ Abdominal bleeding. ${ }^{\mathrm{c}}$ Two abdominal bleedings, one abdominal wall hematoma. ${ }^{\mathrm{d}}$ One gastrointestinal bleeding, one abdominal bleeding.

tive laboratory parameters, there was a significant difference in preoperative weight (heavier in the rLRYGB group, although the BMI was not significantly different), resulting from the differences in indication: 15 (83.3\%) rLRYGB patients were converted because of insufficient weight loss, weight regain, or planned second step; only $3(16.7 \%)$ were revised due to reflux problems or other non-weight associated problems. For rRRYGB, $24(58.5 \%)$ patients were revised due to body weight, with a higher proportion of reflux patients $(n=17$, $41.5 \%)$.

The operative time differed significantly, robotically completed 37 min earlier on average (Table 2). The mean time for docking was $5 \mathrm{~min}$. Postoperative laboratory val- ues did not differ regarding hemoglobin and $\mathrm{WBC}$, but significantly lower CRP values were found on days 1 and 2 with rRRYGB. The inpatient stay after rRRYGB was also significantly shorter. The overall rate of complications (Clavien-Dindo classification II-V) was lower after rRRYGB (7.3\%) than rLRYGB (22.2\%), although not significantly, and there was no significant difference in individual complications. Weight loss tended to be greater with rLRYGB, with a significantly greater reduction in BMI after 30 days.

Further subgroup analyses divided according to the indication for rRYGB (reflux or weight related) yielded comparable results with lower operative times in the two robotic groups as well as reduced postoperative CRP 


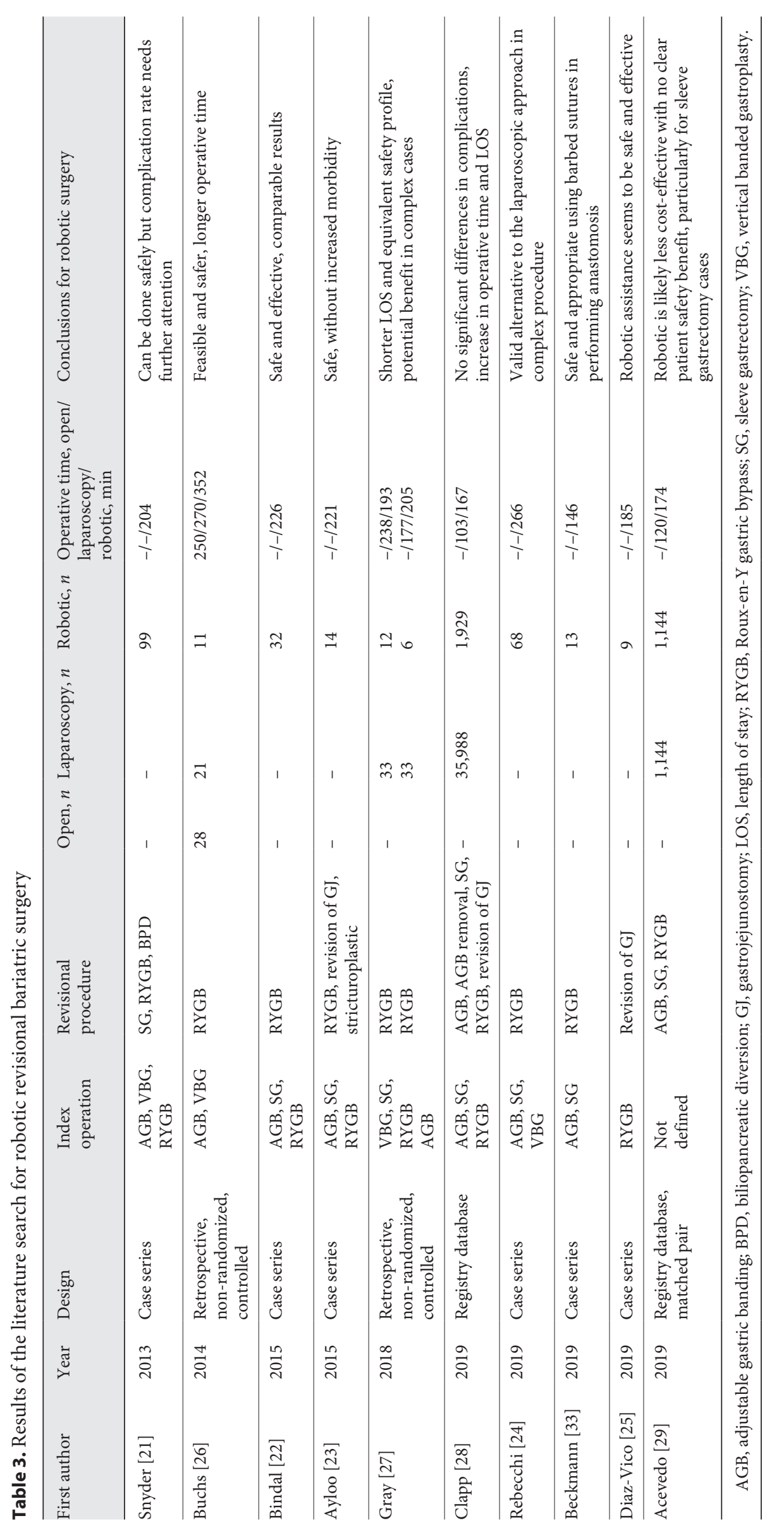


values and LOS (data not shown, online supplementary Table 4).

\section{Discussion}

Complex bariatric rRYGB procedures can be performed safely with the robotic system, with significantly shorter operative times and low complication rates.

The role of the robot in bariatric surgery is still not clearly defined. While performing RSG, the disadvantages may outweigh the advantages [6-8]. The robot seems to be advantageous in primary RYGB regarding postoperative complications $[17,18]$, but at the expense of higher costs and longer operative times [19]. Revision interventions are considered more complex and complicated [34], and the potential advantages of the robot system are likely to become more apparent. The literature indicates that several case series, two controlled non-randomized studies, and an analysis of the MBSAQIP have been published (Table 3), as discussed below.

\section{Pros}

By using the da Vinci Xi System, we achieve significantly shorter operative times compared to rLRYGB surgery. Previous studies mostly reported extended operative times when using the robot $[26,28,29]$, up to $6 \mathrm{~h}$ in one study [26]. It has been argued that the results differ only in operative time and LOS in comparable cohorts, and that no benefit is apparent [28]. However, there was a significantly higher proportion of technical simple gastric band removals in the laparoscopic revision group, while consecutively the relative share of complex RYGB operations was higher in the robot group. One study reported a significant reduction in operative time in a subgroup of rRRYGB (193 min, da Vinci Xi) versus rLRYGB (238 $\mathrm{min}$ ) [27]. We also used the Xi System. With a mean surgical time of $126 \mathrm{~min}$ (rRRYGB), we were well below previous publications (range 167-352 min; Table 3) including MBSAQIP evaluations [28, 29]. The docking time of 5 min no longer plays a significant role with corresponding experience using the system.

Regarding postoperative complications (Clavien-Dindo [31]), there was a lower number of complications with rRRYGB in our study. The difference was not significant due to the low number of cases and incidence of complications. Other groups have also reported low complication and leakage rates (Table 3). In the largest case series of 99 robotic procedures, a high rate of minor complications (17\%) was found, without hemorrhages or leaks as a major complication [21]. Another group concluded that there is no clear benefit with robotic revisional bariatric surgery in terms of patient safety, reporting a higher complication rate after robotic surgery in unmatched rSG [29].
We found significantly lower CRP values on postoperative days 1 and 2 after rRRYGB compared to rLRYGB. The lower CRP levels in the robotic group cannot be solely explained by lower complication rates. The subgroup analysis of all rRYGB patients without complications (Clavien-Dindo 0 and I) also showed significantly lower CRP levels after rRRYGB ( $p=0.00014$, data not shown). We consider this a possible sign of a more precise and atraumatic surgical approach using the robot.

Regarding the LOS, results to date are contradictory. A reduction in the LOS was found in one study using the robot [27], while the registry data showed an extended LOS [28]. In the study by Clapp et al. [28], the clear differences between cohorts regarding index operation and procedure must be mentioned. Our data show a significant reduction in LOS with rRRYGB. Due to the temporal divergence of the two groups, we believe that LOS was influenced by a more stringent patient management in this group.

Apart from well measurable parameters, an additional advantage is the comfort for the surgeon, in particular in patients with a very high BMI. A survey among bariatric surgeons shows a prevalence of $73-88 \%$ of physical complaints caused by laparoscopic bariatric surgery [35]. However, the study is not yet able to provide valid data on robotically active colleagues.

\section{Cons}

Although the steering of the system is intuitive, it is complex. Moreover, haptic feedback is missing. Therefore, simulator training and training on mini pig and human cadavers are essential before using the system [36]. A Proctoring program is available, whereby centers are accompanied by experienced robotic surgeons during their first procedures. The program is recommended to avoid typical pitfalls in the initial phase.

Higher costs hamper a wider use of the system. A detailed analysis of all incurring costs cannot be provided. The average additional costs for the use of the Xi system have already been calculated in other publications and amount to approximately EUR 2,000 [9,37]. The cost of the operating room per minute in our clinic has been estimated at EUR 15. With a reduction in the operative time by $37 \mathrm{~min}$, the remaining additional costs are about EUR 1,450 . It has been discussed to what extent the additional costs are compensated by avoiding complications [38], or whether the investments are amortized by advertising effects [15]. Currently, we do not assume that the use of the robot in bariatric surgery is cost free.

\section{Weaknesses and Strengths}

This study gives a comprehensive overview of revisional bariatric surgery using the robotic da Vinci Xi System against laparoscopy, with a complete evaluation of complications after 30 days. Due to the different indica- 
tions and index surgery, the sample size is small and groups are not comparable in all points. In addition, the laparoscopic surgeries were performed earlier. At the beginning of the study, our cumulative institutional experience was limited to some more than 600 bariatric procedures. On the other hand, the entire learning curve with the Xi System can be found in the robotic group. While the latter benefits the laparoscopic cohort, the overall experience with the procedure gained in the laparoscopic RYGB would favor the robotic cohort. Furthermore, the study cannot offer a complete annual follow-up, as some operations have only recently been performed.

\section{Conclusion}

Retrospective analysis shows that revisional bariatric surgery using a robotic system is safe. Performing rRRYGB results in a low rate of complications. The operative time is shorter in rRRYGB than rLRYGB. Otherwise, results were largely comparable. Structured training and participation in a Proctoring program seem to be reasonable to overcome the learning curve and typical pitfalls. Further studies are necessary to confirm these results.

\section{Acknowledgement}

The manuscript was edited by Deborah Nock (Medical WriteAway, Norwich, UK).

\section{Statement of Ethics}

The study was conducted ethically in accordance with the World Medical Association Declaration of Helsinki and with the approval of the local ethics committee. Informed written consent was obtained from all individual participants included in the study.

\section{Disclosure Statement}

J.H.B., W.S., and T.T. are active as speakers for Johnson \& Johnson Medical. J.H.B., T.T., J.-H.E., and T.B. received grants for training with the da Vinci Xi Surgical System through Intuitive Surgical Sàrl. J.H.B. and J.-H.E. work as proctors for Intuitive Surgical Sàrl. T.B. received the da Vinci ${ }^{\circledR}$ Xi Surgical System from Intuitive. Surgical Sàrl for the purpose of clinical research. A.-S.M., J.-N.K., and M.L. state that they have no conflicts of interest to declare. The authors declare that they have no further competing interests.

\section{Funding Sources}

The robotic procedures were performed on the da Vinci ${ }^{\circledR} \mathrm{Xi}$ Surgical System, provided by Intuitive Surgical Sàrl for the purpose of clinical research to the Department of General, Visceral-, Thoracic-, Transplantation-, and Pediatric Surgery, Kurt-Semm Center for Laparoscopic and Robotic Assisted Surgery, University Hospital Schleswig-Holstein, Campus Kiel, Germany. The services of the medical writer were funded by Intuitive Surgical Sàrl. The funders had no role in study design, data collection and analysis, decision to publish, or preparation of the manuscript.

\section{References}

1 Colquitt JL, Pickett K, Loveman E, Frampton GK. Surgery for weight loss in adults. Cochrane Database Syst Rev. 2014 Aug;(8):CD003641.

2 Mahawar KK, Nimeri A, Adamo M, Borg CM, Singhal R, Khan O, et al. Practices Concerning Revisional Bariatric Surgery: a Survey of 460 Surgeons. Obes Surg. 2018 Sep;28(9):2650-60.

3 Brethauer SA, Kothari S, Sudan R, Williams B, English WJ, Brengman M, et al. Systematic review on reoperative bariatric surgery: American Society for Metabolic and Bariatric Surgery Revision Task Force. Surg Obes Relat Dis. 2014 Sep-Oct;10(5):952-72.

4 AWMF Guideline. Chirurgie der Adipositas und metabolischer Erkrankungen [Internet]. 2018. Available from: https://www.awmf.org/ leitlinien/detail/ll/088-001.html.

5 Coblijn UK, Verveld CJ, van Wagensveld BA, Lagarde SM. Laparoscopic Roux-en-Y gastric bypass or laparoscopic sleeve gastrectomy as revisional procedure after adjustable gastric band-a systematic review. Obes Surg. 2013 Nov;23(11):1899-914.

6 Magouliotis DE, Tasiopoulou VS, Sioka E, Zacharoulis D. Robotic versus Laparoscopic
Sleeve Gastrectomy for Morbid Obesity: a Systematic Review and Meta-analysis. Obes Surg. 2017 Jan;27(1):245-53.

7 Moon RC, Stephenson D, Royall NA, Teixeira AF, Jawad MA. Robot-Assisted Versus Laparoscopic Sleeve Gastrectomy: Learning Curve, Perioperative, and Short-Term Outcomes. Obes Surg. 2016 Oct;26(10):2463-8.

8 Fazl Alizadeh R, Li S, Inaba CS, Dinicu AI, Hinojosa MW, Smith BR, et al. Robotic versus laparoscopic sleeve gastrectomy: a MBSAQIP analysis. Surg Endosc. 2019 Mar;33(3):917-22.

9 Beckmann JH, Aselmann H, Egberts JH, Bernsmeier A, Laudes M, Becker T, et al. Roboterassistierter vs. laparoskopischer Magenbypass. Chirurg. 2018;89(8):612-20.

10 Sanchez BR, Mohr CJ, Morton JM, Safadi BY, Alami RS, Curet MJ. Comparison of totally robotic laparoscopic Roux-en-Y gastric bypass and traditional laparoscopic Roux-en-Y gastric bypass. Surg Obes Relat Dis. 2005 Nov-Dec;1(6):549-54.

11 Ahmad A, Carleton JD, Ahmad ZF, Agarwala A. Laparoscopic versus robotic-assisted Roux-en-Y gastric bypass: a retrospective, single-center study of early perioperative outcomes at a community hospital. Surg Endosc. 2016 Sep;30(9):3792-6.

12 Buchs NC, Morel P, Azagury DE, Jung M, Chassot G, Huber O, et al. Laparoscopic versus robotic Roux-en-Y gastric bypass: lessons and long-term follow-up learned from a large prospective monocentric study. Obes Surg. 2014 Dec;24(12):2031-9.

13 Scozzari G, Rebecchi F, Millo P, Rocchietto S, Allieta R, Morino M. Robot-assisted gastrojejunal anastomosis does not improve the results of the laparoscopic Roux-en-Y gastric bypass. Surg Endosc. 2011 Feb;25(2):597603.

14 Stefanidis D, Bailey SB, Kuwada T, Simms C, Gersin K. Robotic gastric bypass may lead to fewer complications compared with laparoscopy. Surg Endosc. 2018 Feb;32(2):610-6.

15 Senellart P, Saint-Jalmes G, Mfam WS, AbouMrad A. Laparoscopic versus full robotic Roux-en-Y gastric bypass: retrospective, single-center study of the feasibility and shortterm results. J Robot Surg. 2019 Jun;14:291-6. https://doi.org/10.1007/s11701-019-00976-5. 
16 Cahais J, Lupinacci RM, Oberlin O, Goasguen N, Zuber K, Valverde A. Less Morbidity with Robot-Assisted Gastric Bypass Surgery than with Laparoscopic Surgery? Obes Surg. 2019 Feb;29(2):519-25.

17 Li K, Zou J, Tang J, Di J, Han X, Zhang P. Robotic Versus Laparoscopic Bariatric Surgery: a Systematic Review and Meta-Analysis. Obes Surg. 2016 Dec;26(12):3031-44.

18 Economopoulos KP, Theocharidis V, McKenzie TJ, Sergentanis TN, Psaltopoulou T. Robotic vs. Laparoscopic Roux-En-Y Gastric Bypass: a Systematic Review and Meta-Analysis. Obes Surg. 2015 Nov;25(11):2180-9.

19 Lundberg PW, Wolfe S, Seaone J, Stoltzfus J, Claros L, El Chaar M. Robotic gastric bypass is getting better: first results from the Metabolic and Bariatric Surgery Accreditation and Quality Improvement Program. Surg Obes Relat Dis. 2018 Sep;14(9):1240-5.

20 Tian HL, Tian JH, Yang KH, Yi K, Li L. The effects of laparoscopic vs. open gastric bypass for morbid obesity: a systematic review and meta-analysis of randomized controlled trials. Obes Rev. 2011 Apr;12(4):254-60.

21 Snyder B, Wilson T, Woodruff V, Wilson E. Robotically assisted revision of bariatric surgeries is safe and effective to achieve further weight loss. World J Surg. 2013 Nov;37(11): 2569-73.

22 Bindal V, Gonzalez-Heredia R, Elli EF. Outcomes of Robot-Assisted Roux-en-Y Gastric Bypass as a Reoperative Bariatric Procedure. Obes Surg. 2015 Oct;25(10):1810-5.

23 Ayloo SM, Choudhury N. Robotic revisional bariatric surgery: single-surgeon case series. Int J Med Robot. 2015 Sep;11(3):284-9.
24 Rebecchi F, Ugliono E, Allaix ME, Toppino M, Borello A, Morino M. Robotic Roux-en-Y Gastric Bypass as a Revisional Bariatric Procedure: a Single-Center Prospective Cohort Study. Obes Surg. 2019 Aug;30:11-17. https:// doi.org/10.1007/s11695-019-04117-7.

25 Diaz-Vico T, Elli EF. Value of robotic-assisted technique in redo gastrojejunostomy for severe stenosis after gastric bypass. J Robot Surg. 2019 Aug. https://doi.org/10.1007/ s11701-019-01009-x.

26 Buchs NC, Pugin F, Azagury DE, Huber O, Chassot G, Morel P. Robotic revisional bariatric surgery: a comparative study with laparoscopic and open surgery. Int J Med Robot. 2014 Jun;10(2):213-7.

27 Gray KD, Moore MD, Elmously A, Bellorin O, Zarnegar R, Dakin G, et al. Perioperative Outcomes of Laparoscopic and Robotic Revisional Bariatric Surgery in a Complex Patient Population. Obes Surg. 2018 Jul;28(7):18529.

28 Clapp B, Liggett E, Jones R, Lodeiro C, Dodoo $\mathrm{C}$, Tyroch A. Comparison of robotic revisional weight loss surgery and laparoscopic revisional weight loss surgery using the MBSAQIP database. Surg Obes Relat Dis. 2019 Jun; 15(6):909-19.

29 Acevedo E, Mazzei M, Zhao H, Lu X, Edwards MA. Outcomes in conventional laparoscopic versus robotic-assisted revisional bariatric surgery: a retrospective, case-controlled study of the MBSAQIP database. Surg Endosc. 2020 Apr;34(4):1573-84. https://doi.org/10.1007/ s00464-019-06917-5.

30 Sharma AM, Kushner RF. A proposed clinical staging system for obesity. Int J Obes. 2009 Mar;33(3):289-95.
31 Dindo D, Demartines N, Clavien PA. Classification of surgical complications: a new proposal with evaluation in a cohort of 6336 patients and results of a survey. Ann Surg. 2004 Aug;240(2):205-13.

32 Kersebaum IN, Schafmayer C, Ahrens M, Laudes M, Becker T, Beckmann JH. Duodenal intussusception of the remnant stomach after biliopancreatic diversion: a case report. BMC Surg. 2018 Aug;18(1):57.

33 Beckmann JH, Kersebaum JN, von Schönfels W, Becker T, Schafmayer C, Egberts JH. Use of barbed sutures in robotic bariatric bypass surgery: a single-center case series. BMC Surg. 2019 Jul;19(1):97.

34 Zhang L, Tan WH, Chang R, Eagon JC. Perioperative risk and complications of revisional bariatric surgery compared to primary Rouxen-Y gastric bypass. Surg Endosc. 2015 Jun; 29(6):1316-20.

35 AlSabah S, Al Haddad E, Khwaja H. The prevalence of musculoskeletal injuries in bariatric surgeons. Surg Endosc. 2018;33:1818-27. https://doi.org/10.1007/s00464-018-6460-1.

36 Egberts J-HH, Beham A, Ghadimi M. Aufbau eines Roboterprogramms. Zentralbl Chir. 2016 Apr; 141(2): 143-4. https://doi. org/10.1055/s-0042-104068.

37 Beckmann JH, Becker T, Schafmayer C. Roboter-assistierte bariatrische Chirurgie in Deutschland. CHAZ. 2019;20(6):294-8.

38 Hagen ME, Pugin F, Chassot G, Huber O, Buchs $\mathrm{N}$, Iranmanesh $\mathrm{P}$, et al. Reducing cost of surgery by avoiding complications: the model of robotic Roux-en-Y gastric bypass. Obes Surg. 2012 Jan;22(1):52-61. 